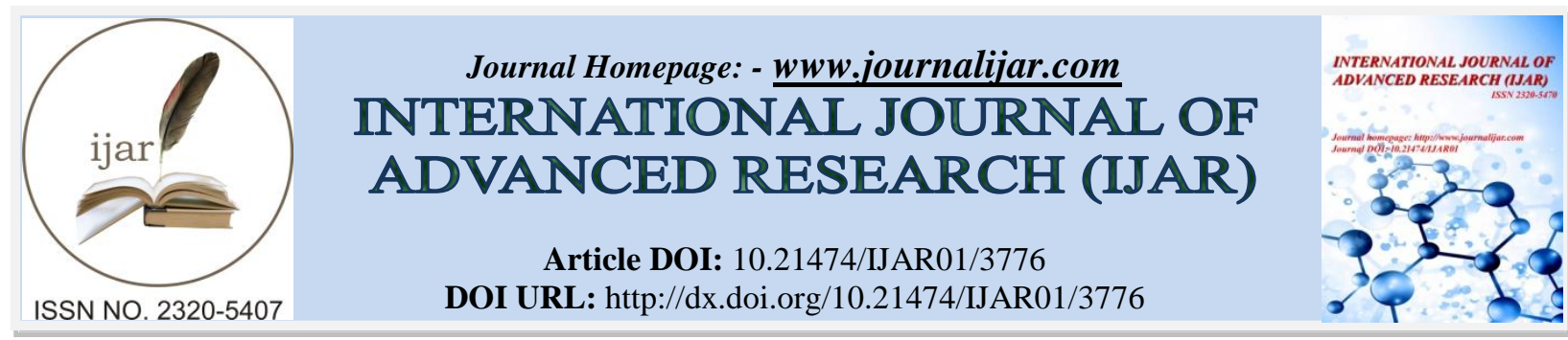

RESEARCH ARTICLE

\title{
COMPARATIVE STUDY BETWEEN CHILDREN WITH INTRACTABLE EPILEPSY ON KETOGENIC DIET AND INTRACTABLE EPILEPTIC CHILDREN ON MEDICATION.
}

\author{
Elham Abdel Ghaffar ${ }^{1}$, Yasmin Gamal El Gendy ${ }^{2}$, Eman R.Abd Almonaem ${ }^{3}$ and Sara Abdel Heady ${ }^{4}$. \\ 1. Professor of Pediatric- Faculty of Medicine - Benha University. \\ 2. Lecturer of Pediatric- Faculty of Medicine - Ain Shams University. \\ 3. Lecturer of Pediatric- Faculty of Medicine - Benha University. \\ 4. Benha Specialized children hospital.
}

\section{Manuscript Info}

n......................

Manuscript History

Received: 03 February 2017

Final Accepted: 01 March 2017

Published: April 2017

Key words:-

ketogenic diet, convulsion, anti-epileptic drugs.

\section{Abstract}

Purpose: to use the regimen of ketogenic diet (modified atkin diet) in addition to anti-epileptic drugs in controlling cases of intractable epilepsy and compare the results with cases receiving anti-epileptic drugs only from one month to 36 months.

Subjects and methods: This is a case control study which was performed on 15 cases of refractory epilepsy received anti-epileptic drugs and follow ketogenic diet (modified atkin diet) which are the cases, and 15 patients with refractory epilepsy received antiepileptic drugs only which are the control with age ranging from 1-36 months and mean age $(1.63 \pm 1.13)$.

Results: showed that, the frequency of convulsions before diet ranged from 2 to 40 with mean 12.4 and standard deviation( SD) 10.769 compared to the frequency of convulsions after diet ranged from 0 to 10 with mean 2.20 and standard deviation 2.95 and there was statistically significant difference between the two groups (P. value was 0.001 ). There was no statistically significant difference between lipid profile of the patients before diet and after diet. Mean \pm standard deviation of Quality Of Life (QOL) scores was $15.71 \pm 1.38$ before diet which changed to $25.57 \pm 3.41$ after diet .This difference was statistically significant (P. value was 0.000$)$.

Conclusion: Modified atkin diet (MAD) improved patients with intractable epilepsy as regards to frequency of convulsions and quality of life.

Copy Right, IJAR, 2017,. All rights reserved.

\section{Introduction:-}

The ketogenic diet is a main stream non pharmacologic therapy that was developed to reproduce the success and remove the limitations of the non-main stream use of fasting to treat epilepsy (Stafstrom and Rho, 2012).

The original Atkins diet is very low in carbohydrates and was developed as a weight reduction therapy. The term 'modified Atkin diet"(MAD) describes the lower carbohydrates limit compared to Atkins recommendations and the emphasis of high fat foods as required on the ketogenic diet (Kossoff et al., 2012). 
The (MAD) was created at Johns Hopkins Hospital to offer a less restrictive dietary treatment.

MAD limits carbohydrates and emphasis protein and fat intake for weight loss.

MAD is easier to follow, requiring no calorie or fluid restrictions, although it does limit carbohydrate intake to around $15 \mathrm{~g}$ per day. Allowed breakfast foods include high-fat meats such as bacon, sausage and ham, as well as eggs and cheese. Low- carbohydrates breads may be allowed in small amounts, as long as the total carbohydrate intake remains within the prescribed levels (Freeman et al., 2007).

On the ketogenic diet, carbohydrates are restricted and so cannot provide for all the metabolic needs of the body. Instead, fatty acids are used as the major source of fuel, These are used through fatty-acid oxidation in the cell's mitochondria (the energy producing part of the cell).Humans can convert some amino acids into glucose by a process called gluconeogenesis ,but cannot do this for fatty acids (Hartman et al ., 2007 ).

Most individuals with epilepsy can successfully control their seizures with medications. However, 20-30\% fail to achieve such control despite trying a number of different drugs (Kossoff, 2009).For this group, and for children in particular, the diet has found a role in epilepsy management (Freemanetal., 2007).

\section{Subjects and Methods:-}

This is a case control prospective study which was performed on 15 cases of refractory "intractable "epilepsy with age ranging from1-36 months and mean age (1.63 \pm 1.13$)$ received anti-epileptic drugs and follow ketogenic diet (modified atkin diet) which are the cases, and 15 patients with refractory epilepsy received anti-epileptic drugs only which are the control. They were 6 males and 9 females (the cases) and the same for the control. They were collected from Outpatient clinics and pediatric departments from Ain Shams university hospital and Benha university Hospital from December 2015 till December 2016.

\section{Inclusion criteria:-} Intractable epilepsy:-

* Inadequate seizure control despite a trial of 2 or 3 anti-epileptic drugs (AED) that are suitable for the type of epilepsy and have been appropriately prescribed at maximum tolerated doses (Berg, 2004).

* Or adequate seizure control with unacceptable drug-related side effects (Goetal., 2008)

\section{Exclusion criteria:}

1. Carnitine deficiency (primary).

2. Carnitine palmitoyl transferase (CPT) 1 or 2 deficiency.

3. Carnitine translocase deficiency.

4. B-oxidation defects.

5. Medium-chain acyl dehydrogenase deficiency (MCAD).

6. Long-chain acyl dehydrogenase deficiency (LCAD).

7. Short-chain acyl dehydrogenase deficiency (SCAD).

8. Porphyria.

9. Pyruvate carboxylase deficiency.

\section{Methods:-}

Patients were subjected to the following before initiation of the diet:-

1. Full history taking with special emphasis on:

Types of convulsions , onset of convulsions, frequency of convulsions, duration of convulsions, what increase what decrease the convulsions, types and number of drugs used, doses, compliance, side effects noticed and it is frequency.

2. Full detailed examination.

3. Laboratory investigations :

- Serum lipid profile: (cholesterol, triglycerides, LDL, VLDL and HDL)

- $\quad$ ABG (Blood gases)

- liver function tests

- $\quad$ kidney function tests 
- $\quad$ serum ammonia

\section{Statistical Analysis:-}

The data were coded, entered and processed on computer using SPSS (version 18). The results were represented in tabular and diagrammatic forms then interpreted. Mean, standard deviation, range, frequency, and percentage were used as descriptive statistics.

The following test was done:

- Student's-test was used to assess the statistical significance of the difference between two populations means in a study involving independent samples.

- Student's paired t-test was used to assess the statistical significance of the difference between two populations means in a study involving paired samples.

$P$ value was considered significant as the following:-

* $\mathrm{P}>0.05$ : Non significant

$* \mathrm{P} \leq 0.05:$ Significant

\section{Results:-}

Table (1) shows that, the frequency of convulsions before diet ranged from 2 to 40 with mean 12.4 and standard deviation 10.769 compared to the frequency of convulsions after diet ranged from 0 to 10 with mean 2.20 and standard deviation 2.95 and there was statistically significant difference between the two groups (P. value was $0.001)$.

Table (2) shows the Severity according to the Chalfont score Before diet ranged from 13 to 30 with mean 20.93 and standard deviation 4.75 compared to the Severity Chalfont score After diet ranged from 0 to 24 with mean 8.53 and standard deviation 7.818 and there was statistically significant difference between the two groups (P. value was $<0.001$ ).

Table (3) shows that, there was no statistically significant difference between lipid profile of the patients before diet and after diet.

Table (4) shows that, the mean \pm standard deviation of Quality Of Life (QOL) scores was $15.71 \pm 1.38$ before diet which changed to $25.57 \pm 3.41$ after diet and this difference was statistically significant.

Table 1:- Comparison between the initial and final frequency of convulsions of the studied patients using paired ttest.

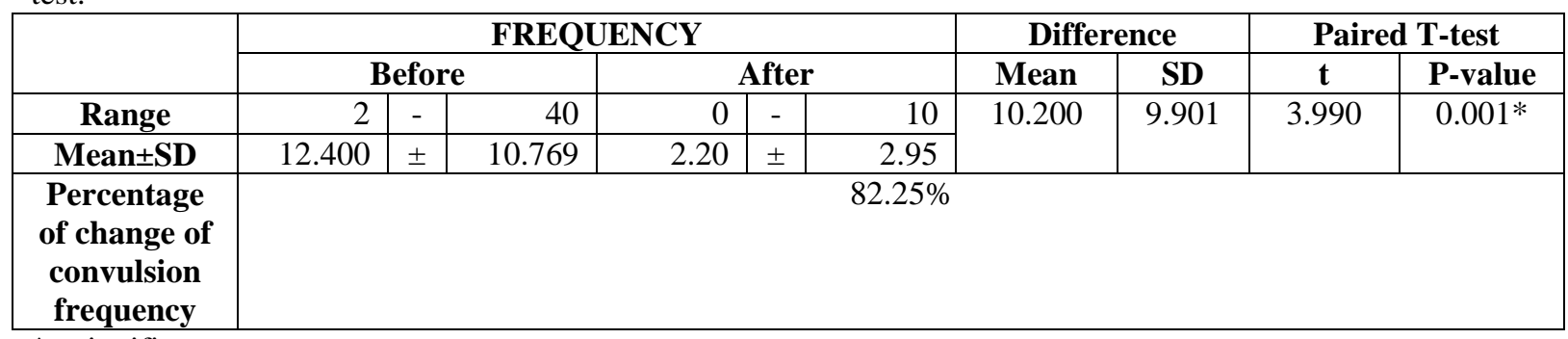

$*=$ significant

Table 2:- Comparison between the severity according to chalfont score before and after diet of the studied patients using paired t-test.

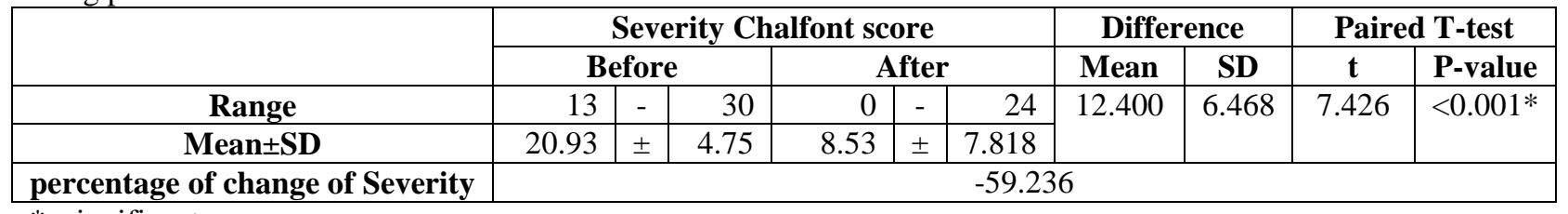

$*=$ significant 
Table 3:- Comparison between the initial and final lipid profile items of the studied patients using paired t-test.

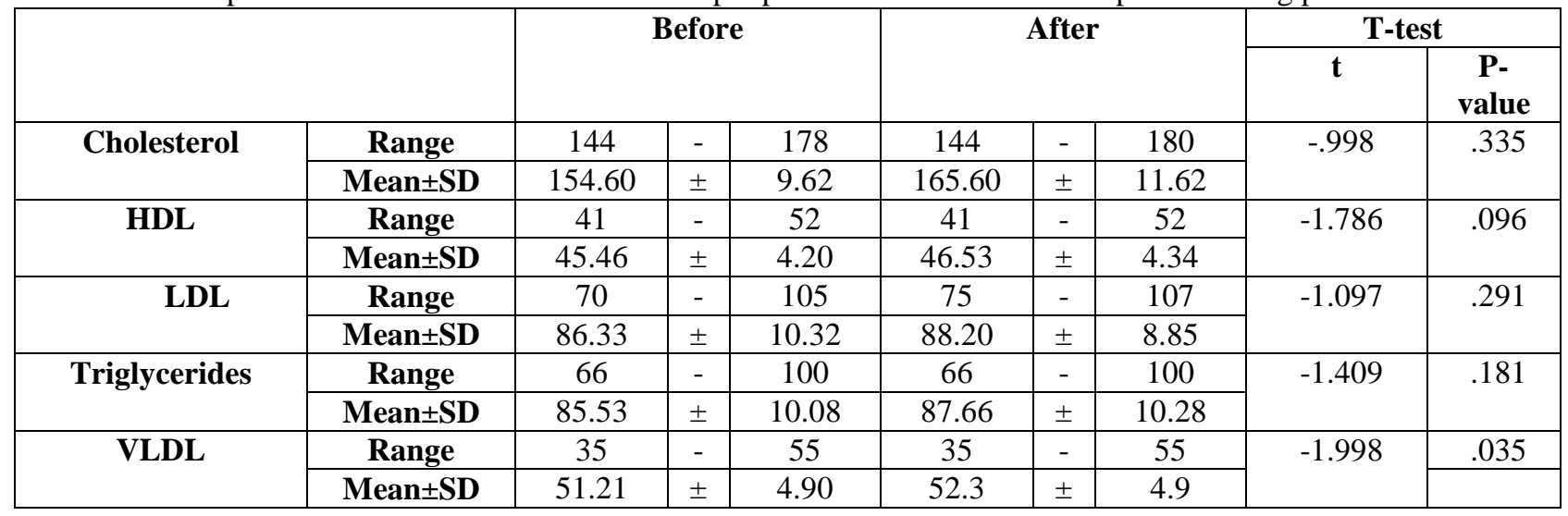

Table 4:- Comparison between initial and final Quality Of Life (QOL) scores using paired t-test.

\begin{tabular}{|c|c|c|c|c|c|c|c|c|}
\hline & \multicolumn{3}{|c|}{ Before } & \multicolumn{3}{c|}{ After } & \multicolumn{3}{c|}{ T-test } \\
\cline { 3 - 9 } & \multicolumn{3}{|c|}{ t } & P-value \\
\hline QOL mean \pm SD & 15.71 & \pm & 1.38 & 25.57 & \pm & 3.41 & -9.994 & 0.000 \\
\hline
\end{tabular}

\section{Discussion:-}

Refractory epilepsy can be defined as inadequate seizure control despite appropriate medical therapy with 2 or 3 AEDs or more in maximally tolerated doses for 18 months to 2 years or adequate seizure control with unacceptable drug-related side effects (Go, and Snead 2008).

The ketogenic diet is a mainstream, non-pharmacologic therapy that was developed to reproduce the success and remove the limitations of the non-mainstream use of fasting to treat epilepsy. Although popular in 1920 and 1930, it was largely abandoned in favor of new anti convulsant drugs (Freeman et al., 2007). Most individuals with epilepsy can successfully control their seizures with medication. However, 20-30\% fail to achieve such control despite trying a number of different drugs. For this group, and for children in particular, the diet has once again found a role in epilepsy management (Kossoff et al., 2009.

The ketogenic diet (KD) is an established non pharmacologic therapy for children with intractable epilepsy (Kossoff, 2004). It has been used worldwide for the treatment of intractable childhood epilepsy (Kossoff and McGrogan, 2005).

The original Atkins diet is very low in carbohydrates and was developed as a weight reduction therapy. The term 'modified Atkin diet (MAD) describes the lower carbohydrate limit compared to Atkins recommendations and the emphasis of high fat foods as required on the ketogenic diet (Kossoff et al., 2012).

The (MAD) was created at Johns Hopkins Hospital to offer a less restrictive dietary treatment. MAD limits carbohydrates and emphasis protein and fat intake for weight loss.

MAD is easier to follow, requiring no calorie or fluid restrictions, although it does limit carbohydrate intake to around $15 \mathrm{~g}$ per day. Allowed breakfast foods include high-fat meats such as bacon, sausage and ham, as well as eggs and cheese. Low- carbohydrates breads may be allowed in small amounts, as long as the total carbohydrate intake remains within the prescribed levels (Freeman et al.,2007).

The modified Atkins diet (MAD) induces ketosis, but without fluid, caloric, or protein restriction, nor the requirement for fasting, food weighing, or hospitalization (Kossoff et al., 2007 and Kang et al., 2007)

Also, MAD induces a state of ketosis by providing a high fat content and few carbohydrates, so it may control seizures by a mechanism similar to that of the KD (Stafstrom, 2004). 
This study showed that, the frequency of convulsions before diet ranged from 2 to 40 with mean 12.4 and standard deviation 10.769 compared to the frequency of convulsions after diet ranged from 0 to 10 with mean 2.20 and standard deviation 2.95 and there was statistically significant difference between the two groups (P. value was 0.001).

This is in agreement with El-Rashidy et al.(2013) who found that there was a decrease in frequency of seizures in 6 of 15 patients on modified atkin diet (MAD), also this is in agreement with the study done in 2010 by Mirjavadi et $\boldsymbol{a l}$., who treated 66 intractable epilepsy children with MAD and found that after 3 months, 12 patients (12.2\%) were seizure free and 39 patients $(59 \%)$ showed $50 \%$ or more reduction in seizure frequency. Our results are in concordance with Karimzadeh et al.(2011) who treated 26 "children with intractable epilepsy" with KD 4:1 (fat: carbohydrates) and found that at the end of the third month, acceptable decrease in seizure frequency (at least 50\%) had occurred in $87 \%$ of children, of which $39 \%$ had complete seizure control. At the end of the sixth month, $63 \%$ of the patients were seizure free. Also Squeal.(2013) in alarge study done on 317 Chinese children showed $>50 \%$ seizure reduction after intake of MAD.

This study showed that, the Severity Chalfont score Before diet ranged from 13 to 30 with mean 20.93 and standard deviation 4.75 compared to the Severity Chalfont score After diet ranged from 0 to 24 with mean 8.53 and standard deviation 7.818 and there was statistically significant difference between the two groups $(\mathrm{P}$. value was $<0.001)$.

Concerning lipid profile of the patients (cholesterol, triglycerides, high density lipoproteins, low density lipoproteins and very low density lipoproteins) there was no significant difference in the lipid profile before and after MAD. This is in agreement with Danish study performed by Maria et al.(2016) in which patients with refractory epilepsy received MAD showed no significant increase in free cholesterol, triglycerides, LDL, HDL and VLDL.

On the other hand, other study reported that there was elevation in "serum cholesterol levels, serum triglycerides levels, serum LDL levels and decrease in serum HDL levels" by using classic solid KD (Fenton et al.,2009).

This study showed that the mean \pm standard deviation of Quality Of Life (QOL) scores was $15.71 \pm 1.38$ before diet which changed to $25.57 \pm 3.41$ after diet .This difference was statistically significant (P. value was 0.000$)$.

\section{Conclusion:-}

Ketogenic diet (modified atkin diet " MAD") improved patients with intractable epilepsy as regard to frequency of convulsions and quality of life.

\section{References:-}

1. El-Rashidy OF, Nassar MF, Abdel-Hamid IA, Shatla RH, Abdel-Hamid MH, Gabr SS, et al.(2009) Modified Atkins diet vs classic ketogenic formula in intractable epilepsy. ActaNeurol Scand. 2013;128:402408.

2. Fenton, C., Chee, C.M., Bergqvist, A.G.C., (2009): Manipulation of types of fats and cholesterol intake can successfully improve the lipid profile while maintaining the efficacy of the ketogenic diet. Infant Child Adolesc. Nutr. 1, 338-341.

3. Freeman JM, Vining EP, Pillas DJ, Pyzik PL, Casey JC, Kelly LM. (2007): The efficacy of the ketogenic diet-2007: A prospective evaluation of intervention in 150 children. Pediatrics. 2007;102:1358-63.

4. Go C., Snead C, (2008): Pharmacologically Intractable Epilepsy In Children: Diagnosis and Preoperative Evaluation. Neurosurg Focus. 2008;25(3):E2

5. Kang HC, Lee HS, You SJ, Kang DC, Ko TS, Kim HD. (2007): Use of a modified Atkins diet in intractable childhood epilepsy. Epilepsia 4S:1S2-1S6.

6. Kossoff EH, (2009): Ketogenic diets: an update for child neurologists. J Child Neurol.; 24(S):979-SS.

7. Kossoff EH, McGrogan JR. (2005): Worldwide use of the ketogenic diet. Epilepsia.; $46: 2$ SO.

8. Kossoff EH, Zupec-Kania BA, Rho JM. (2009):Ketogenic diets: an update for child neurologists. J Child Neurol.; 24(S):979-SS.

9. María J. Alberti, M. Agustinho A, and Argumedo L., (2016) Recommendations for the clinical management of children with refractory epilepsy receiving the ketogenic dietArch Argent Pediatr 2016;114(1):56-63 / 56 
10. Mirjavadi SA, Tonekaboni SH, Ghazavi M, Azargash BE, Abdallah GF, Ghofrani M (2010): Iran journal child neurology vol4; no2.

11. Stafstrom CE, Rho JM.(2012): The Ketogenic Diet as a Treatment Paradigm for Diverse Neurological Disorders. Frontiers in Pharmacology. 2012;3:59. doi:10.3389/fphar.2012.00059.

12. Stafstrom CE. (2004): An introduction to seizures and epilepsy. In: Stafstrom CE, Rho JM, editors. Epilepsy and the ketogenic diet. Totowa: Humana Press;. ISBN 1-5SS29-295-9.

13. Suo C, Liao J, Lu X, Fang K, Hu Y, Chen L, et al. (2013): Efficacy and safety of the ketogenic diet in Chinese children. Seizure. 2013;22:174-8. 